\title{
The neglected biliary mucus and its phosphatidylcholine content: a putative player in pathogenesis of primary cholangitis -a narrative review article
}

\author{
Wolfgang Stremmel ${ }^{1 \wedge}$, Martina Lukasova $^{2}$, Ralf Weiskirchen ${ }^{3} \wedge$ \\ ${ }^{1}$ Medical Center Baden-Baden, Baden-Baden, Germany; ${ }^{2}$ Pharmacy of University Clinics of Heidelberg, Heidelberg, Germany; ${ }^{3}$ Institute of \\ Molecular Pathobiochemistry, Experimental Gene Therapy and Clinical Chemistry, RWTH University Hospital Aachen, Aachen, Germany \\ Contributions: (I) Conception and design: All authors; (II) Administrative support: None; (III) Provision of study materials or patients: None; (IV) \\ Collection and assembly of data: All authors; (V) Data analysis and interpretation: All authors; (VI) Manuscript writing: All authors; (VII) Final \\ approval of manuscript: All authors. \\ Correspondence to: Wolfgang Stremmel. Medical Center Baden-Baden, Beethovenstr. 2/76530 Baden-Baden, Germany. \\ Email: wolfgangstremmel@aol.com.
}

\begin{abstract}
Primary sclerosing cholangitis (PSC) is a rare progressive cholangitis resulting in cirrhosis and cholangiocellular carcinoma. The pathogenesis is unclear and an effective medical therapy is not available. It is highly associated to ulcerative colitis for which recently a disturbance of the tight junction (TJ) barrier has been claimed as etiologic feature. Genetic mouse models with intestinal TJ disruption showed a defective transport of phosphatidylcholine (PC) to intestinal mucus. Consequently, an ulcerative colitis phenotype developed. In the present study we evaluate whether there is also a paracellular transport of PC through TJ to the apical side of cholangiocytes. As in ulcerative colitis, a TJ defect could lead to deficient PC in biliary mucus. It would impair the protective barrier against aggressive bile acids in bile. Indeed with polarized biliary tumor cells a vectorial transport of PC from basal to luminal side was demonstrated using a transwell culture system. PC was not taken up by the cells but moved paracellularly via TJ to the apical side driven by luminal $\mathrm{HCO}_{3}$ - generated by the Cystic Fibrosis Transmembrane Conductance Regulator (CFTR) and the anion exchange protein 2 (AE2). If such a TJ-mediated PC translocation to the apical surface of cholangiocytes could be disrupted in a genetic mouse model, a PSC phenotype would be expected. With such an experimental model functional operative therapies can be evaluated. We propose that disruption of TJ mediated paracellular transport of PC to the apical side of cholangiocytes could lead to biliary mucus PC depletion. This may be a pathogenetic factor for development of PSC.
\end{abstract}

Keywords: Primary sclerosing cholangitis (PSC); ulcerative colitis; phosphatidylcholine (PC); tight junction barrier (TJ barrier)

Submitted Feb 27, 2020. Accepted for publication Jul 08, 2020.

doi: $10.21037 /$ atm-20-3591

View this article at: http://dx.doi.org/10.21037/atm-20-3591

^ ORCID: Wolfgang Stremmel, 0000-0002-8545-1753; Ralf Weiskirchen, 0000-0003-3888-0931. 


\section{Introduction}

The pathogenesis of PSC is unknown and there is no medical therapy available. Therefore, the prognosis without liver transplantation is limited. Ulcerative colitis and PSC are epidemiologically linked. We reviewed the evidence of a possible common pathogenetic background. This could have implication for new therapeutic approaches.

We present the following article in accordance with the NARRATIVE REVIEW reporting checklist (available at http://dx.doi.org/10.21037/atm-20-3591).

\section{Methods}

The literature was combed through via PubMed according to the listed keywords. We considered German and English original publications (basic science and clinical), reviews and abstracts of the last 30 years.

\section{Epidemiology}

Primary sclerosing cholangitis (PSC) is a chronic progressive disease of unknown pathogenesis leading from inflammation of the biliary tree to sclerosing cholangitis and finally to biliary cirrhosis and eventually to cholangiocellular carcinoma (1). It occurs with a frequency of 10 cases/100,000 inhabitants and manifests predominantly in males (70\%) between $30-40$ years of age. However, numbers vary from country to country with a higher incidence and prevalence in Northern European countries. The fact that about $5 \%$ of patients with ulcerative colitis develop PSC explains why most of PSC patients $(70 \%)$ have concomitant ulcerative colitis $(1,2)$. Both diseases do not necessarily appear at the same time and in some cases the cholangitis occurs years before ulcerative colitis. The colitis in PSC patients is predominant in the right colon with an increased risk of colon cancer. Even after liver transplantation the occurrence of ulcerative colitis has been reported (1).

\section{Clinical presentation}

Patients may be asymptomatic or complain as in other cholestatic conditions about fatigue (6\%) and pruritus (10\%) (3). Further symptoms are pain in the upper right abdomen due to an enlarged liver (44\%), in the later cirrhotic state patients reveal splenomegaly (39\%), jaundice (6\%), weight loss and episodes of fever. An overlap with autoimmune hepatitis is observed in $35 \%$ of children and $5 \%$ of adults. About threefourth of patients develop gallstones and bile duct stones. Osteoporosis occurs as also in other chronic cholestatic diseases.

\section{Natural course of PSC}

From the asymptomatic state patients develop over an unpredictable time frame clinical symptoms (4). At advanced disease stage with a sclerosing cholangitis phenotype, bacterial infections of the biliary tree occur episodically which enhance disease progression and require antibiotic therapy (3). Cirrhosis with portal hypertension and their respective complications implicate end stage liver disease. In this situation liver failure gradually develops. There is an increased risk for development of cholangiocarcinoma (400fold higher risk as in the general population, cumulative 30 -year incidence $20 \%$ ), even at that young age of patients. Therefore, the prognosis is unfortunate (4).

\section{Diagnosis}

Laboratory evaluation shows elevated cholestatic enzymes, e.g., alkaline phosphatase (AP) and gamma glutamyltransferase (GGT) as well as later also conjugated hyperbilirubinemia. In $50 \%$ of patients $\operatorname{IgM}$ and/or autoantibodies [antinuclear antibodies (ANA), antismooth muscle antibodies (ASMA), anti-neutrophil cytoplasmatic antibodies (ANCA)] and in $10 \%$ IgG4 are elevated. The latter of which is associated with a more progressive course often responding to steroid treatment $(3,5)$. The involvement of liver parenchyma leads to elevated transaminases (ALT and AST). Liver function can be monitored by determination of cholinesterase and measurement the international normalized ratio (INR) as well as creatinine, reflecting concomitant renal failure. The Model for End-stage Liver Disease (MELD) score reflects the urgency for liver transplantation (3). However, some parameters of liver dysfunction are less rapidly progressing in comparison to the potential danger to develop cholangiocarcinoma with infaust prognosis. Therefore, an exceptional MELD score is practiced to provide the patients a fair chance to survive (3).

Ultrasound imaging of the liver is rather unspecific revealing some enhanced reflexes at the biliary tree and an increased echogenicity of the enlarged liver in precirrhotic 


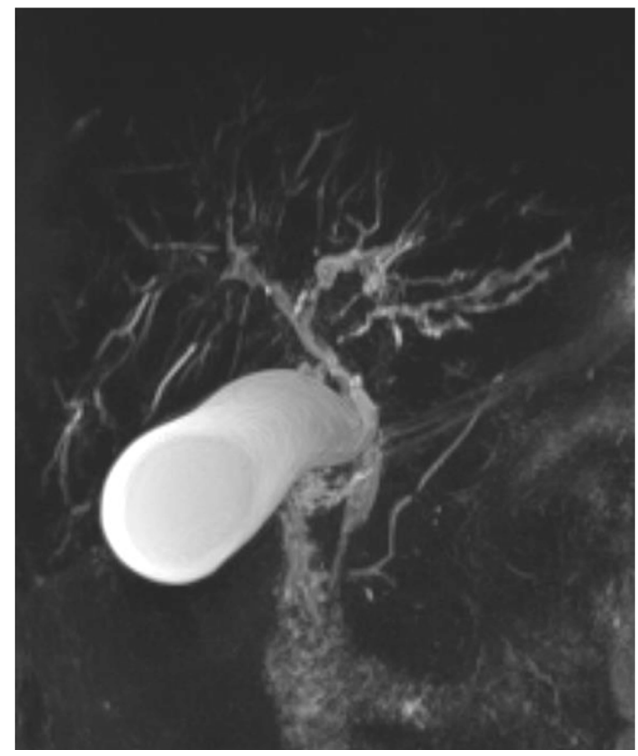

Figure $1 \mathrm{MRCP}$ picture of a representative patient with PSC. It reveals diffuse annular, segmental stricture formation and duct irregularities throughout the biliary tract and prestenosal dilatation of the lumen, here predominantly in the left lateral lobe. Typical is the "pruned tree" appearance. (Courtesy of Dr. T. Weber, University Hospital of Heidelberg). MRCP, magnetic resonance cholangiopancreatography; PSC, primary sclerosing cholangitis

patients. Leading to diagnosis is a magnetic resonance image (MRI) of the biliary system (i.e., magnetic resonance cholangiopancreatography, MRCP) (6), which shows moniliform, multifocal stenoses or sometimes single localized stenoses down to the papilla (Figure 1). The earlier employed endoscopic cholangiopancreatography (ERCP) bears the risk of ascending cholangitis induced by this procedure and is therefore less favorable. However, it is employed when stricture biopsy or cytology, sampling of bile for microbiological analysis, or the dilatation of critical stenoses are required (7). As more invasive diagnostic procedure a liver biopsy (in case of coagulation impairment a transjugar biopsy) with histologic evaluation can be employed, particularly in small duct PSC (8). The evidence of cholangitis and peribiliary sclerosis with onion shaped appearance is pathognomonic (Figure 2) (8). A cirrhotic pattern can also be identified. However, due to a minimal risk of biopsy it may not be necessary when the diagnosis is highly probable by the other parameters. In case a mass is seen by imaging, a fine needle biopsy is indicated to verify the nature of the lesion.

\section{Treatment}

A causal therapy is not available due to lack of knowledge about pathogenesis. Therefore, only symptomatic therapies as medication or endoscopic interventions are applied (Table 1).

The previous often used ursodeoxycholate was shown not to be effective $(3,9)$. For pruritus bile acid sequestrants, e.g., cholestyramine, are used sometimes in combination with antihistamines to ease the urge to scratch. In severe cases opioid, e.g., naltrexone, can be an option $(10,11)$. Obeticholic acid represents another new promising approach (1).

For treatment of biliary infection bile accessible antibiotics have to be provided, possibly after preceding microbial testing of endoscopically acquired bile. Preventive broad-spectrum antibiotics are also recommended for endoscopic procedure at the biliary system (12).

In case of deficiency of fat soluble vitamins a substitution therapy has to be considered. For the frequently observed osteoporosis (13) the application of vitamin D is recommended (14).

In case of endoscopic accessible, high grade stenoses a balloon dilation via endoscopic retrograde cholangiopancreatography (ERCP) should be performed. It was shown to improve the clinical course of the disease (15-19).

The last option is liver transplantation which has to be considered in this patient population rather early $(3,7)$. In most cases it cures PSC, but it is also reported to recur in the transplanted liver (20). A concomitant ulcerative colitis will still persist or may even develop.

In summary for this disease with poor prognosis no satisfying, long term medical therapy is available. Endoscopic therapy is of significant benefit for the patient. Most patients are cured by liver transplantation (Table 1).

\section{Hypothesis towards pathogenesis of PSC}

It is questionable whether it is predominantly an immune system-mediated disease because immunosuppressive therapies were not successful $(1,3)$.

There is evidence that it is genetically determined: common phenotype and natural course, young to medium aged adults, closely associated to ulcerative colitis (1).

\section{The phosphatidylcholine (PC) transport story}

Recently in intestine we discovered a new transport route for PC from systemic sources (lipoproteins) through the 

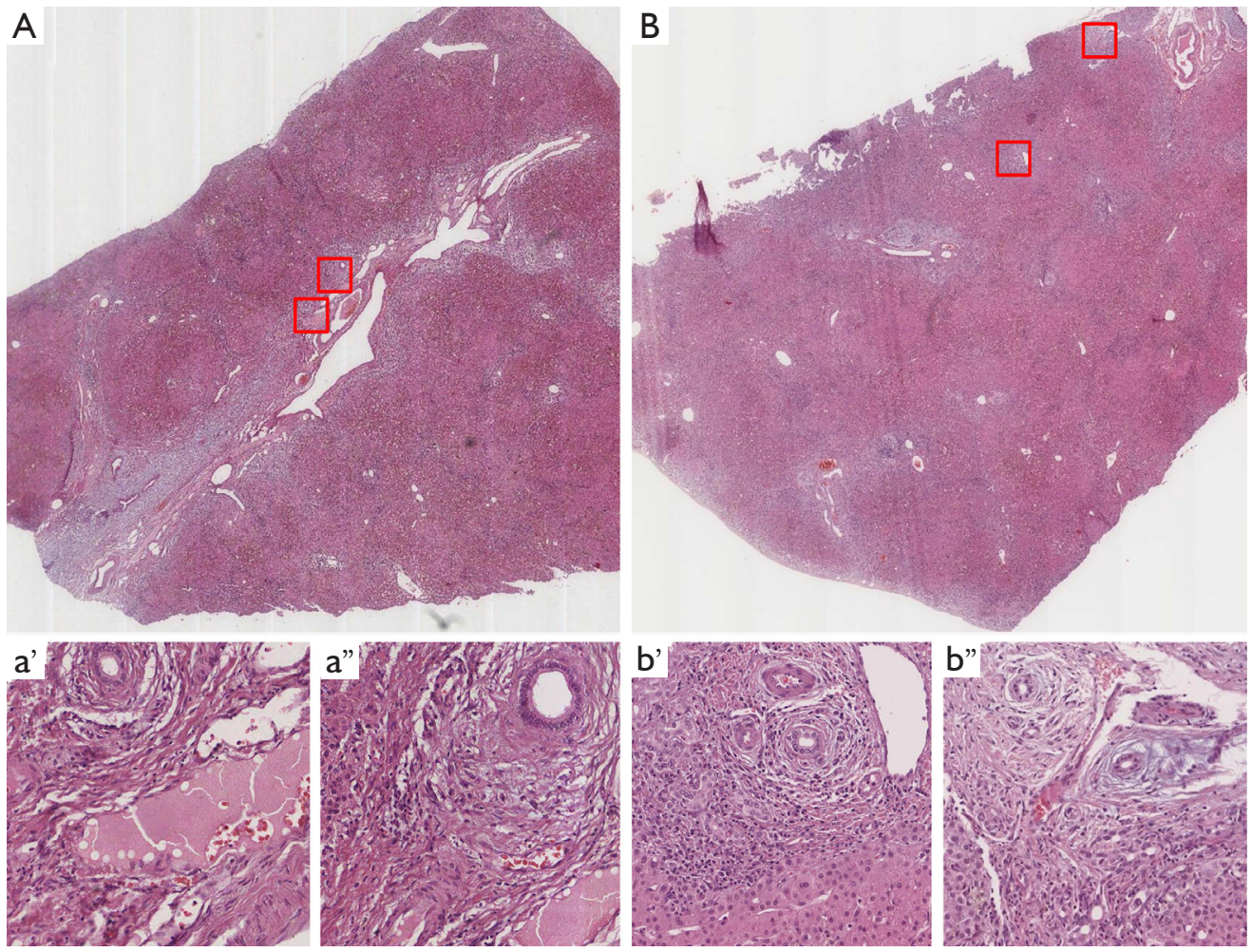

Figure 2 Liver section obtained from two representative patients (A,B) with primary sclerosing cholangitis. Typical features of PSC patients are small and intermediate bile ducts surrounded by fibrous tissue that can be seen at higher magnifications (a', a", b', b"). Note the cuboidal shape of cholangiocytes which is compatible with the notion of loosening of intercellular adhesion due to a tight junction defect. (Courtesy of the Aachen students Pathology archives). PSC, primary sclerosing cholangitis.

Table 1 Therapy targets of PSC

\begin{tabular}{ll}
\hline Symptom or sign & Therapy \\
\hline Disease progression & No available therapy \\
Itching & Cholestyramine, obeticholic acid \\
Bacterial cholangitis & Antibiotics \\
Leading stenosis & Balloon dilatation \\
Imminent risk of \\
cholangiocarcinoma
\end{tabular}

PSC, primary sclerosing cholangitis.

intercellular spaces within the mucosa and passage across the tight junction (TJ) barrier towards the luminal surface (21). Here it binds to the membrane bound mucin 3 from where it is handled to mucin 2 to establish a hydrophobic mucus barrier against the microbiota in colonic lumen (21). Driving force for the non-apolipoprotein bound amphiphilic PC across the $\mathrm{TJ}$ is the luminal accumulation of $\mathrm{HCO}_{3}{ }^{-}$.
This negative luminal charge at the apical side of mucosal cells is generated by the Cystic Fibrosis Transmembrane Conductance Regulator (CFTR) and the Anion Exchange protein 2 (AE2).

This paracellular, TJ mediated transport route of PC is rather unusual because it was always assumed that TJs represent barrier function allowing only passage of water and electrolyte. In case of TJ disruption, it was suspected that luminal microbiota, toxins, carcinogens or antigens could pass to the system.

In the following we summarize the results revealing in vivo evidence for the PC apical transport route, pathophysiologic consequences of its disturbance and therapeutic options.

It was evaluated in a genetic colitis mouse model with exclusive TJ disruption in the gut by intestinal specific knockout of the TJ adaptor proteins kindlin 1 and 2 (22). Here the same structural and functional alterations were observed as in human ulcerative colitis: (I) due to loss of the 
intercellular attachment the cylindrical and closely attached mucosal cell layer turned into cuboidal shaped cells with distended crypt Lumina (disturbed crypt architecture). (II) Functionally PC did not pass to the luminal mucus, the mucus PC concentration dropped and its hydrophobicity was impaired. This lack of protection resulted in invasion of bacteria into the submucosa (22). Since the gene deletion was intestine-specific, the liver remained normal as expected. A secondary induced PSC caused by bacterial translocation from gut seemed therefore also to be excluded. In human ulcerative colitis-even in remission-the mucus PC is by $70 \%$ reduced $(23,24)$. Thus, it is an intrinsic disposition of pathogenetic significance. Human ulcerative colitis was shown also to be caused by TJ disturbance (22).

The knockout mice developed an ulcerative colitis phenotype (22). For compensation of the genetically caused lack of PC, a topical substitution from the luminal side was considered, because the physiologic route from systemic lipoproteins was blocked by TJ knockout. In this setting an excess of PC was supplemented orally by deep tube feeding allowing PC to pass to distal parts of the intestine for incorporation into the mucus. Indeed the ulcerative colitis phenotype could be prevented (22). It is claimed to be accomplished by symptomatic filling of empty PC stores within the mucus (binding to mucin 2) to reestablish a hydrophobic barrier. This simple procedure could also be a therapeutic or preventive strategy to fight ulcerative colitis in man. For this purpose, PC was coated with a polymer for delayed release in colon. Certainly, four randomized controlled trials (RCT) showed the efficacy of this approach (25-30). The missing PC in mucus of ulcerative colitis patients could be replenished (25). Within 5-7 weeks, the patients markedly improved in regard to clinical activity, endoscopic appearance and histology. Indeed, most patients achieved clinical remission. A further maintenance of remission observation confirmed the beneficial effect of delayed release PC application in ulcerative colitis (29). Unfortunately, a phase III trial did not show the expected improvement to induce remission (31). This was in part due to the protocol which required simultaneous application of mesalazine together with PC $v s$. mesalazine alone instead of placebo without mesalazine (32). The reason was that otherwise the control group would not receive any therapy despite disease activity. However, experimentally it could be shown that due to the detergent effect of mesalazine an intraluminal foam with PC is generated with suboptimal access to the mucosal surface (33). A maintenance of remission trial was performed with patients from the preceding induction trial. Due to early stop of this induction study, the remission trial did not receive sufficient participants. In this 3 -arms trial, placebo, mesalazine and PC were provided as single medication. Indeed PC was better as placebo and similar effective as mesalazine (34). However, the number of patients was too low to allow a statistically significant finding. In further contrast to the successful initial three RCT $(25,26,28)$, the later RCT used gastric acid resistant PC formulations instead of intestinally released preparations $(30,31)$.

\section{The link between ulcerative colitis and PSC}

Due to the fact that ulcerative colitis and PSC commonly associate and PSC reveals a non-explainable chronic inflammation of the biliary epithelium, it was hypothesized that both diseases share a common denominator.

The biliary epithelium is considered as a mucosal surface which as all other mucosal surfaces produces protective mucus to seal the surface (35). It exerts hydrophobicity by enrichment of PC bound to mucins which are locally produced (36). According to our findings in intestinal mucosa, the PC apically located in the adherent mucus of the biliary epithelium could originate from systemic sources via passage across the TJ barrier $(21,37)$. In case of a potential lack of PC in the biliary mucus as reason for PSC manifestation, the supplementation from the luminal biliary side seemed mechanistically impossible. It is not accessible for the mucus despite the fact that PC is highly abundant in bile by hepatocellular secretion via the ATP-binding cassette (ABC) transporter protein MDR3 (Multidrug Resistance Protein 3, ABCB4) at the canalicular plasma membrane. At the same location the very efficient bile-salt export pump (BSEP, ABCB11) releases bile acids to build in a strong association with PC as micellar solution in bile (38).

This micelle-incorporated PC cannot be utilized for incorporation into biliary mucus. In the experimental situation when a knockout mouse with lack of the mouse equivalent to MDR3, the mdr2 (ABCB4) was generated, a severe cholangitis phenotype resembling human PSC appeared (39). The explanation is that the free bile acids without secreted PC can attack the mucus PC for its removal into bile, thus, thinning the hydrophobic protective barrier and inducing inflammation.

This pathomechanism does not apply to PSC because 
hepatic PC secretion via canalicular membrane localized MDR3 is intact and the analysis of bile did not reveal a deficiency of PC in PSC patients (40). However, the attached biliary epithelial mucus compartment with a low PC content could be a potential focus of interest, although-to our knowledge-this has never been considered before. Another important feature of this mucus compartment is its capability to accumulate $\mathrm{HCO}_{3}{ }^{-}$via the biliary epithelial AE2 and CFTR which establishes a functional protective bicarbonate umbrella $(41,42)$. In this context it is of interest that for paracellular TJ mediated transport of $\mathrm{PC}$ a high apical $\mathrm{HCO}_{3}^{-}$is required $(21,37)$. Accordingly, the working hypothesis was generated that PC in mucus may pass paracellularly along the lateral side of cholangiocytes through the TJ barrier to the apical side.

\section{Experimental prove of the $\mathbf{T} \mathbf{J}$ mediated transport mechanism in biliary epithelium}

We refer to experiments performed with the biliary tumor cell line Mz-ChA-1 grown for 21 days to confluency in Transwell culture systems (37). This cell line is the best model with cholangiocyte characteristics. Primary cholangiocytes are not available for tissue culture experiments. Kinetic analyses were performed with the polarized cell layer by application of PC and other substrates in a transwell culture system to either the basal or apical compartment and translocation to the counter compartment was analyzed. Intracellular recovery was negligible. The principle of a vectoral transport to the apical compartment was proven when the equilibrium distribution was examined after application of $100 \mathrm{mM}$ PC to the basal and apical side. In contrast to the also non-cell-permeable inulin, an enrichment at the apical and depletion at the basal side was noted (Figure $3 A$ ). The data prove a paracellular movement of $\mathrm{PC}$ to the luminal side of cholangiocytes.

This transport was specific for choline-containing phospholipids (PC, lyso-PC and sphingomyelin) but not for other phospholipids, cholesterol and fatty acids (Figure 3B).

A negative apical charge stimulated, whereas a positive charge prevented the apical translocation (Figure 3C). As responsible charge carrier CFTR and AE2 were identified because respective siRNA treatment of the cells inhibited transport accordingly. By this procedure also the involvement of TJ was verified as downregulation of claudin-1, -2, -4 and -8 , Zonula Occludens-1 (Z01), kindlin-1 and -2 as well as chemical disruption of TJ inhibited PC translocation from the basal side (Figure 3D) (37).
In addition, apical supplementation with mucin 1 or 2 or even taurocholate stimulated as PC acceptor molecules the transport to the apical side (Figure 3E).

The data presented in this study lead to the hypothesis that PC originates from lipoproteins in the circulation. It passes through the intercellular space between biliary epithelial cells to the TJ from where they jump to the luminal side to be bound to mucins for generation of a protective mucus shield against bile acids and infection (Figure 4). For functional prove of the concept in vivo, we attempt as next step to generate a genetic mouse model with specific deletion of TJ in biliary epithelial cells.

If in this model a cholangitis phenotype can be reproduced it would be a clue to pathogenesis of PSC. The biliary tree should show a lack of PC in the biliary mucus compartment, biliary epithelium destruction caused by bile salt attack, possibly bacterial invasion, submucosal inflammation and fibrous tissue for repair.

After availability of a suitable animal model for PSC, therapeutic strategies can be developed. In clinical trials the value of ursodeoxycholate application was extensively examined $(3,9)$. It was shown not to be effective in reversing the course of the disease which, according to our hypothesis, is also not expected. To induce PC secretion across the biliary epithelium would be the ultimate goal. Whether it is achievable via oral application of excess PC is doubtful but should be examined under the hypothesis that a high systemic PC load may push more PC across the biliary epithelium.

It would be a therapeutic challenge to target PC to the biliary lumen without being captured by bile acids. Since aggressive bile acids attacking the mucus barrier may represent the final executer of biliary epithelium injury, it could be an approach to exchange those against more mucus protective bile acids (43). NorUDCA with enhanced cholehepatic shunting showed in initial trials in PSC promising results (44) although its mucus protective mode of action has not been addressed yet. Since they undergo cholehepatic shunting, i.e., uptake by cholangiocytes, they have to leave behind PC. Theoretically this PC released from mixed micelles is available to biliary mucus for filling empty PC binding sites. This could restore its protective barrier function and reverse damage to the biliary epithelium.

\section{Discussion}

This review collects arguments for a completely new 
A

Equilibrium distribution of phosphatidylcholine versus inulin after equal application of $100 \mathrm{mM}$ to the apical and basal compartment in the transwell system

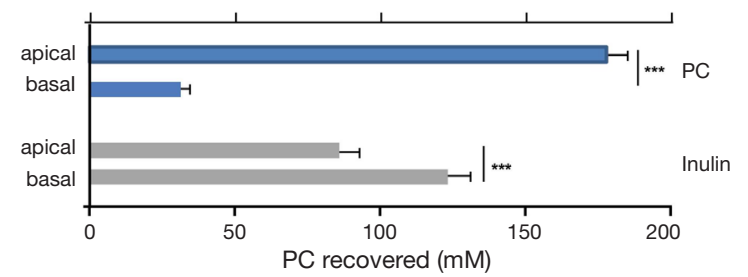

B

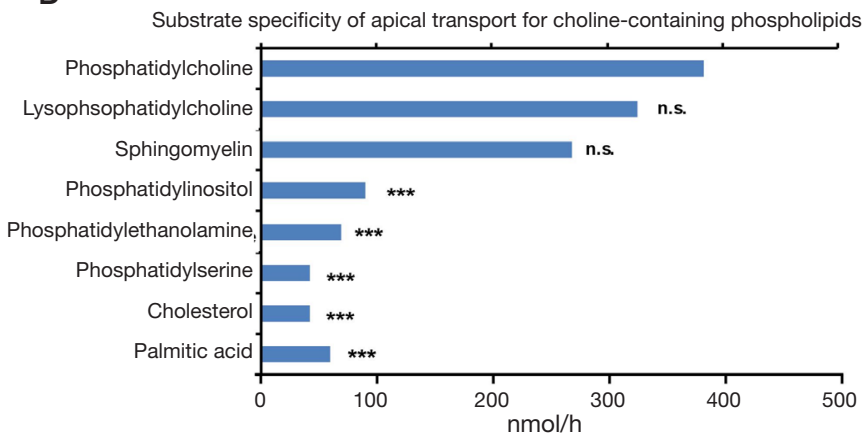

$\mathrm{D}$
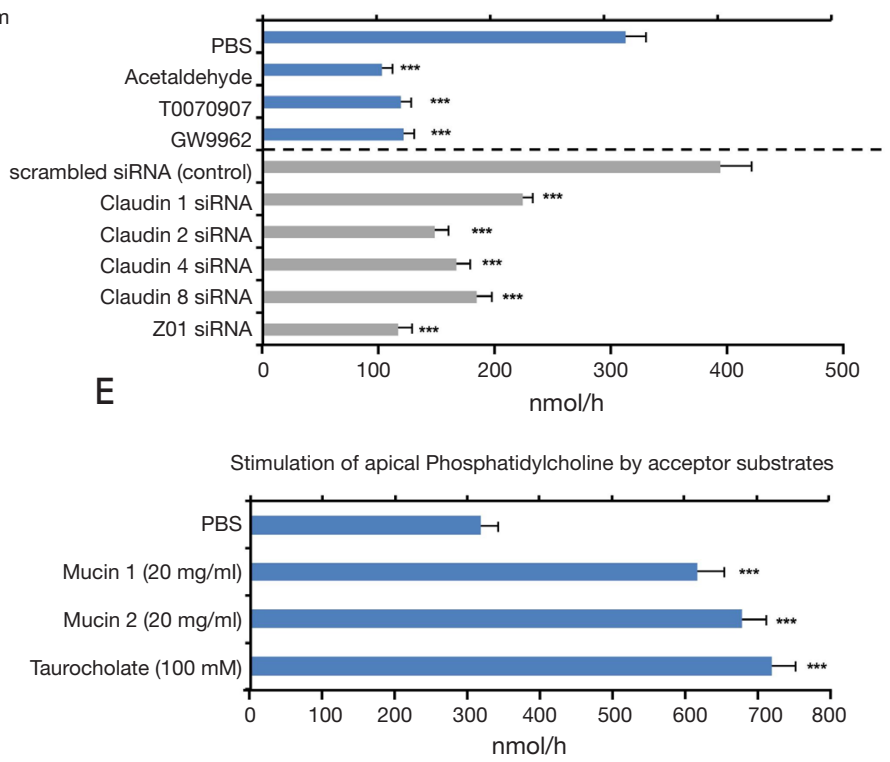

C

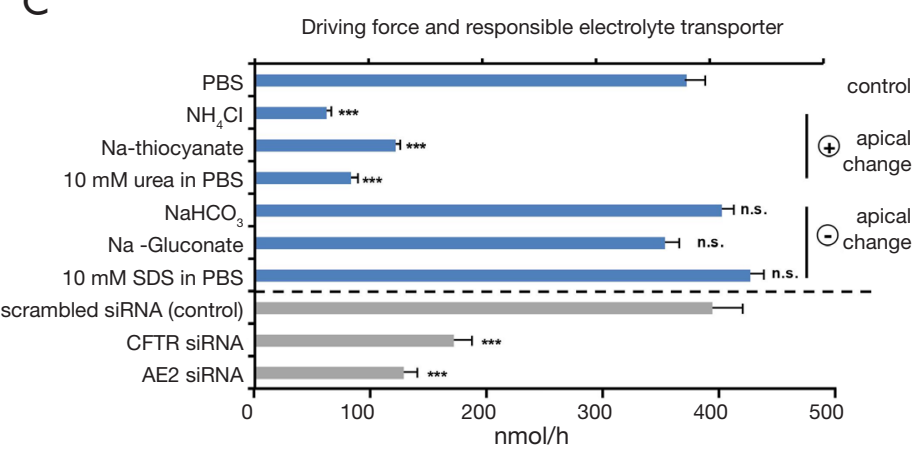

Figure 3 Transport of PC evaluated with polarized biliary epithelial cells grown in a transwell tissue culture system. Transport data were obtained after $1 \mathrm{~h}$ exposure to the basal side (21). (A) Equilibrium distribution of PC versus inulin when applied equally at $100 \mathrm{mM}$ to the apical and basal compartment. Over $1 \mathrm{~h}$ incubation vectoral transport of PC was directed apically devoiding PC at the basal side. In contrast, for inulin a significant higher accumulation at the basal side was registered. (B) Substrate specificity. Apical transport was examined for $1 \mathrm{~h}$ after basal application of the different substrates at $10 \mathrm{mM}$ concentration. (C) Mechanistics of apical PC translocation after basal application $(10 \mathrm{mM})$. Ionic driving forces were generated by apical application of salts and substrates where anionic and cationic partners permeate the membrane at different velocities, thus leaving at the apical cell surface a positive or negative-charged environment. The involvement of the responsible anion exporters CFTR and AE2 was proven by their downregulation using respective siRNAs in comparison to scrambled siRNA. PC $(10 \mathrm{mM})$ was applied to the basal compartment. (D) Prove of tight junction (TJ) involvement in apical transport of PC after their chemical disruption by ACA vapor or the indicated PPAR- $\gamma$ inhibitors T0070907 and GW9962. The involvement of the responsible TJ proteins was evaluated by indicated siRNA preincubation in comparison to scrambled siRNA. PC (10 mM PC) was applied to the basal compartment in polarized Mz-ChA-1 cells. (E) Enhancement of apical PC transport by apical application of mucin $1(20 \mathrm{mg} / \mathrm{mL}), \mathrm{mucin} 2$ $(20 \mathrm{mg} / \mathrm{mL})$ and taurocholate $(100 \mathrm{mM})$ as luminal acceptor substances. PC $(10 \mathrm{mM})$ was applied to the basal compartment. Means \pm SD, $\mathrm{n}=6$. Significances were calculated against PBS (control); ${ }^{* *}, \mathrm{P}<0.001$. PC, phosphatidylcholine; CFTR, Cystic Fibrosis Transmembrane Conductance Regulator; AE2, Anion Exchange protein 2; ACA, acetaldehyde; PPAR- $\gamma$, peroxisome proliferator activated receptor gamma. 

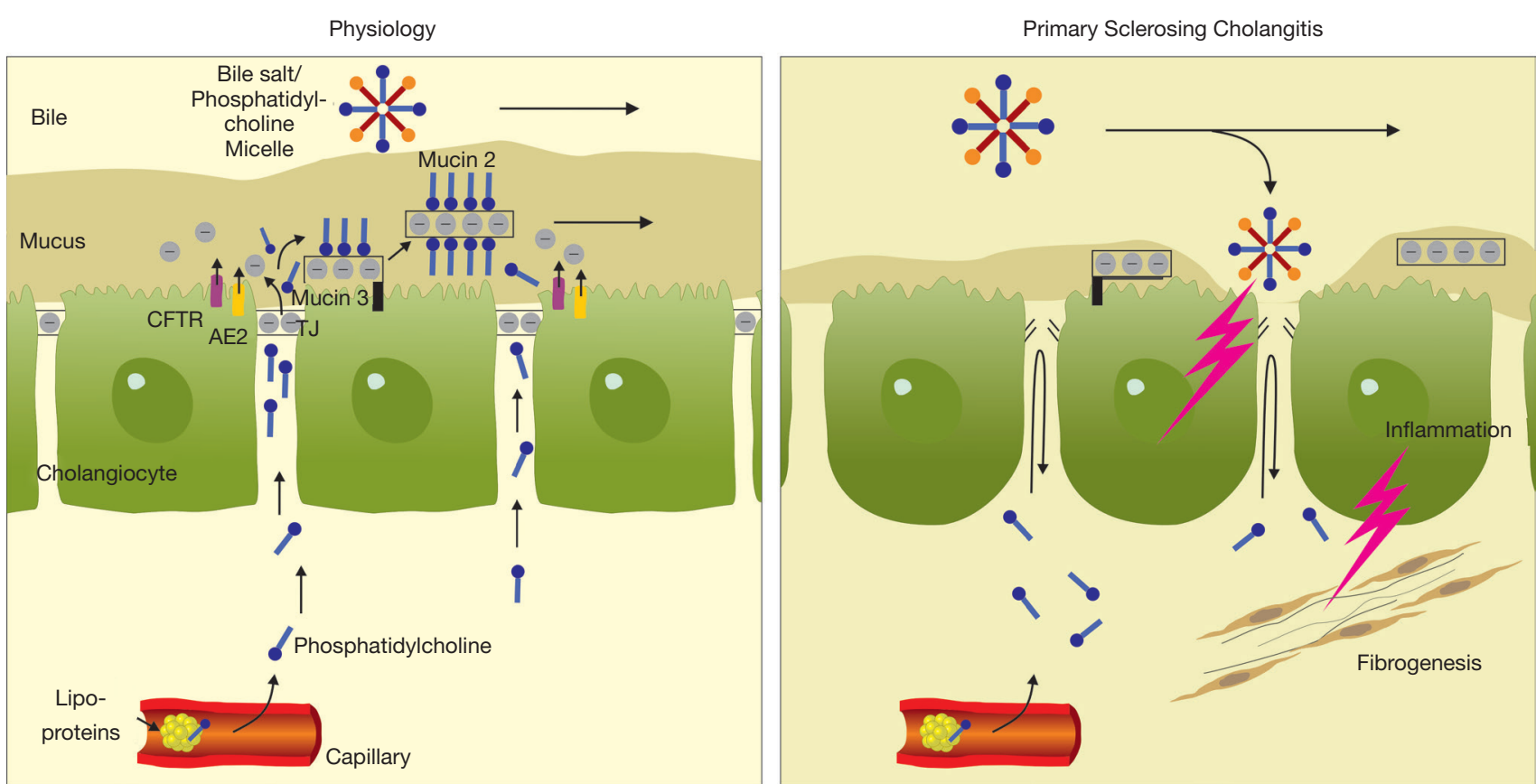

Figure 4 Hypothesis to physiology and pathophysiology of PC transport at the cholangiocyte epithelium in PSC. Illustrated is the transport of PC from systemic sources to the mucus compartment of the biliary epithelium across the tight junction barrier. It is driven by negative apical charge. The apically transported PC is initially bound to membrane anchored mucin 3 from where it is handled to secretory mucin 2 for distal movement along the biliary tree. In PSC the PC transport is prohibited by genetically determined disruption of the tight junction barrier, consequently cholangiocytes develop a cuboidal shape. Luminal aggressive bile acids destroy cholangiocytes due to absence of the protective mucus PC shield, leading to inflammation and consequent fibrosis. CFTR, Cystic Fibrosis Transmembrane Conductance Regulator; AE2, Anion Exchange protein 2; PC, phosphatidylcholine; PSC, primary sclerosing cholangitis.

hypothesis explaining the cause of PSC. It is based on the epidemiologic link between ulcerative colitis and PSC. As a common denominator we present the characteristic feature of intestinal and biliary epithelia to translocate PC paracellularly across the TJ barrier. PC originates from lipoproteins within the circulation and passes to the biliary mucus compartment. This space between the epithelial cell layer and the biliary lumen has never been considered to be of any pathogenetic significance. The PC bound to mucins could function as hydrophobic barrier against the biliary lumen. The conclusion is highly speculative: an unusual hypothesis and scare experimental data. The basic research of PC translocation across a polarized monolayer of the biliary tumor cell line Mz-ChA-1 is the only relevant experimental evidence (37). However, there are many publications available to $\mathrm{PC}$ translocation in intestine and its disturbance as pathogenetic factor for development of ulcerative colitis (21-30). Based on this, in colitis new therapeutic strategies were proposed and tested in clinical trials. The shortcoming is, that it comes basically only from our research group. Since one has to start, we used this review to stimulate the consideration of the biliary mucus compartment as a relevant pathogenetic factor. The theory behind this is simple to comprehend and apparently not completely beside the point. However, it has to face a scientific community with different views. Therefore, we have to prove our hypothesis now. It is one of our future goals to support our hypothesis with in vivo studies. For this purpose, we want to generate a genetic mouse model with a biliary epithelium specific deletion of TJs. We expect a reduction of the mucus PC content and development of cholangitis. This will be compared with human PSC. mostly by structural/histological staining analyses.

\section{Summary}

The discovery of the pathogenesis of PSC remains a challenge. Its resolution could open new avenues for therapy of this devastating disorder.

A clue to pathogenesis could be the disturbance of 
paracellular translocation of $\mathrm{PC}$ to the apical surface of the epithelium. By binding of PC to mucins it serves to establish a protective mucus shield towards luminal bile acids. A genetically determined lack of biliary mucus PC could be a pathogenetic contributor. If verified, therapy could reverse the manifestation by measures to increase the PC concentration in this compartment or ameliorate the aggressive properties of bile acids towards PC binding.

\section{Acknowledgments}

Funding: None.

\section{Footnote}

Provenance and Peer Review: This article was commissioned by the Guest Editors (Ralf Weiskirchen and Wolfgang Stremmel) for the series "Unresolved Basic Issues in Hepatology" published in Annals of Translational Medicine. The article was sent for external peer review organized by the Guest Editors and the editorial office.

Reporting Checklist: The authors have completed the NARRATIVE REVIEW reporting checklist. Available at http://dx.doi.org/10.21037/atm-20-3591

Peer Review File: Available at http://dx.doi.org/10.21037/ atm-20-3591

Conflicts of Interest: All authors have completed the ICMJE uniform disclosure form (available at http://dx.doi. org/10.21037/atm-20-3591). The series "Unresolved Basic Issues in Hepatology" was commissioned by the editorial office without any funding or sponsorship. Ralf Weiskirchen and Wolfgang Stremmel served as the unpaid Guest Editors of the series. Ralf Weiskirchen serves as an unpaid editorial board member of Annals of Translational Medicine from Aug 2018 to Jul 2020. The authors have no other conflicts of interest to declare.

Ethical Statement: The authors are accountable for all aspects of the work in ensuring that questions related to the accuracy or integrity of any part of the work are appropriately investigated and resolved.

Open Access Statement: This is an Open Access article distributed in accordance with the Creative Commons Attribution-NonCommercial-NoDerivs 4.0 International
License (CC BY-NC-ND 4.0), which permits the noncommercial replication and distribution of the article with the strict proviso that no changes or edits are made and the original work is properly cited (including links to both the formal publication through the relevant DOI and the license). See: https://creativecommons.org/licenses/by-nc-nd/4.0/.

\section{References}

1. Karlsen TH, Folseraas T, Thorburn D, et al. Primary sclerosing cholangitis - a comprehensive review. J Hepatol 2017;67:1298-323.

2. Khosravi Khorashad A, Khajedaluee M, Mokhtari Amirmajdi E, et al. Frequency and risk factors of primary sclerosing cholangitis among patients with inflammatory bowel disease in North-East of Iran. Gastroenterol Hepatol Bed Bench 2015;8:200-6.

3. Lindor KD, Kowdley KV, Harrison ME, et al. ACG Clinical Guideline: Primary Sclerosing Cholangitis. Am J Gastroenterol 2015;110:646-59; quiz 60.

4. Takakura WR, Tabibian JH, Bowlus CL. The evolution of natural history of primary sclerosing cholangitis. Curr Opin Gastroenterol 2017;33:71-7.

5. Mendes FD, Jorgensen R, Keach J, et al. Elevated serum IgG4 concentration in patients with primary sclerosing cholangitis. Am J Gastroenterol 2006;101:2070-5.

6. Berstad AE, Aabakken L, Smith HJ, et al. Diagnostic accuracy of magnetic resonance and endoscopic retrograde cholangiography in primary sclerosing cholangitis. Clin Gastroenterol Hepatol 2006;4:514-20.

7. Goldberg D, Bittermann T, Makar G. Lack of standardization in exception points for patients with primary sclerosing cholangitis and bacterial cholangitis. Am J Transplant 2012;12:1603-9.

8. Burak KW, Angulo P, Lindor KD. Is there a role for liver biopsy in primary sclerosing cholangitis? Am J Gastroenterol 2003;98:1155-8.

9. Lindström L, Hultcrantz R, Boberg KM, et al. Association between reduced levels of alkaline phosphatase and survival times of patients with primary sclerosing cholangitis. Clin Gastroenterol Hepatol 2013;11:841-6.

10. Bunchorntavakul C, Reddy KR. Pruritus in chronic cholestatic liver disease. Clin Liver Dis 2012;16:331-46.

11. Imam MH, Gossard AA, Sinakos E, et al. Pathogenesis and management of pruritus in cholestatic liver disease. J Gastroenterol Hepatol 2012;27:1150-8.

12. Rupp C, Bode K, Weiss KH, et al. Microbiological Assessment of Bile and Corresponding Antibiotic 
Treatment: A Strobe-Compliant Observational Study of 1401 Endoscopic Retrograde Cholangiographies. Medicine (Baltimore) 2016;95:e2390.

13. Gasser RW. Cholestatis and metabolic bone disease - a clinical review. Wien Med Wochenschr 2008;158:553-7.

14. Herlong HF, Recker RR, Maddrey WC. Bone disease in primary biliary cirrhosis: histologic features and response to 25-hydroxyvitamin D. Gastroenterology 1982;83:103-8.

15. Stiehl A. Primary sclerosing cholangitis: the role of endoscopic therapy. Semin Liver Dis 2006;26:62-8.

16. Johnson GK, Geenen JE, Venu RP, et al. Endoscopic treatment of biliary tract strictures in sclerosing cholangitis: a larger series and recommendations for treatment. Gastrointest Endosc 1991;37:38-43.

17. Johnson GK, Saeian K, Geenen JE. Primary sclerosing cholangitis treated by endoscopic biliary dilation: review and long-term follow-up evaluation. Curr Gastroenterol Rep 2006;8:147-55.

18. Lee JG, Schutz SM, England RE, et al. Endoscopic therapy of sclerosing cholangitis. Hepatology 1995;21:661-7.

19. Kaya M, Petersen BT, Angulo P, et al. Balloon dilation compared to stenting of dominant strictures in primary sclerosing cholangitis. Am J Gastroenterol 2001;96:1059-66.

20. Campsen J, Zimmerman MA, Trotter JF, et al. Clinically recurrent primary sclerosing cholangitis following liver transplantation: a time course. Liver Transpl 2008;14:181-5.

21. Stremmel W, Staffer S, Gan-Schreier H, et al.

Phosphatidylcholine passes through lateral tight junctions for paracellular transport to the apical side of the polarized intestinal tumor cell-line CaCo2. Biochim Biophys Acta 2016;1861:1161-9.

22. Stremmel W, Staffer S, Schneider MJ, et al. Genetic Mouse Models with Intestinal-Specific Tight Junction Deletion Resemble an Ulcerative Colitis Phenotype. J Crohns Colitis 2017;11:1247-57.

23. Ehehalt R, Wagenblast J, Erben G, et al. Phosphatidylcholine and lysophosphatidylcholine in intestinal mucus of ulcerative colitis patients. A quantitative approach by nanoElectrospray-tandem mass spectrometry. Scand J Gastroenterol 2004;39:737-42.

24. Braun A, Treede I, Gotthardt D, et al. Alterations of phospholipid concentration and species composition of the intestinal mucus barrier in ulcerative colitis: a clue to pathogenesis. Inflamm Bowel Dis 2009;15:1705-20.

25. Stremmel W, Merle U, Zahn A, et al. Retarded release phosphatidylcholine benefits patients with chronic active ulcerative colitis. Gut 2005;54:966-71.

26. Stremmel W, Ehehalt R, Autschbach F, et al. Phosphatidylcholine for steroid-refractory chronic ulcerative colitis: a randomized trial. Ann Intern Med 2007;147:603-10.

27. Stremmel W, Hanemann A, Braun A, et al. Delayed release phosphatidylcholine as new therapeutic drug for ulcerative colitis---a review of three clinical trials. Expert Opin Investig Drugs 2010;19:1623-30.

28. Stremmel W, Braun A, Hanemann A, et al. Delayed release phosphatidylcholine in chronic-active ulcerative colitis: a randomized, double-blinded, dose finding study. J Clin Gastroenterol 2010;44:e101-7.

29. Stremmel W, Hanemann A, Ehehalt R, et al. Phosphatidylcholine (lecithin) and the mucus layer: Evidence of therapeutic efficacy in ulcerative colitis? Dig Dis 2010;28:490-6.

30. Karner M, Kocjan A, Stein J, et al. First multicenter study of modified release phosphatidylcholine "LT-02" in ulcerative colitis: a randomized, placebo-controlled trial in mesalazine-refractory courses. Am J Gastroenterol 2014;109:1041-51.

31. Dignass A, Schnabel R, Romatowski J, et al. Efficacy and safety of a novel high-dose mesalazine tablet in mild to moderate active ulcerative colitis: a doubleblind, multicentre, randomised trial. United European Gastroenterol J 2018;6:138-47.

32. Falk P. Phosphatidylcholine (LT-02) for induction of remission in ulcerative colitis. Identifier No NCT 02142725 2017. Available online: https://clinicaltrials.gov/ ct2/show/NCT02142725

33. Stremmel W, Staffer S, Gehrke S. The Detergent Effect of Mesalazine Interferes with Phosphatidylcholine Binding to Mucin 2. Inflamm Intest Dis 2019;3:107-15.

34. Dignass A, Stremmel W, Bilianskyi L, et al. Efficacy and safety of gastro-resistant phosphatidylcholine (LT-02) for maintenance of remission in patients with ulcerative colitis initially refractory to mesalazine: a randomised, doubleblind, double-dummy placebo-controlled study (PCG-4). United Europ Gastroenterology J 2018;6:A76.

35. Johansson ME, Sjövall H, Hansson GC. The gastrointestinal mucus system in health and disease. Nat Rev Gastroenterol Hepatol 2013;10:352-61.

36. Lichtenberger LM. The hydrophobic barrier properties of gastrointestinal mucus. Annu Rev Physiol 1995;57:565-83.

37. Stremmel W, Staffer S, Weiskirchen R. Phosphatidylcholine Passes by Paracellular Transport to the Apical Side of the Polarized Biliary Tumor Cell Line 
Mz-ChA-1. Int J Mol Sci 2019;20:4034.

38. Dawson PA, Lan T, Rao A. Bile acid transporters. J Lipid Res 2009;50:2340-57.

39. Smit JJ, Schinkel AH, Oude Elferink RP, et al. Homozygous disruption of the murine mdr2 P-glycoprotein gene leads to a complete absence of phospholipid from bile and to liver disease. Cell 1993;75:451-62.

40. Gauss A, Ehehalt R, Lehmann WD, et al. Biliary phosphatidylcholine and lysophosphatidylcholine profiles in sclerosing cholangitis. World J Gastroenterol 2013;19:5454-63.

41. Hohenester S, Wenniger LM, Paulusma CC, et al.

Cite this article as: Stremmel W, Lukasova M, Weiskirchen R. The neglected biliary mucus and its phosphatidylcholine content: a putative player in pathogenesis of primary cholangitisa narrative review article. Ann Transl Med 2021;9(8):738. doi: 10.21037/atm-20-3591
A biliary $\mathrm{HCO} 3-$ umbrella constitutes a protective mechanism against bile acid-induced injury in human cholangiocytes. Hepatology 2012;55:173-83.

42. Banales JM, Prieto J, Medina JF. Cholangiocyte anion exchange and biliary bicarbonate excretion. World $\mathrm{J}$ Gastroenterol 2006;12:3496-511.

43. Beuers U, Kullak-Ublick GA, Pusl T, et al. Medical treatment of primary sclerosing cholangitis: a role for novel bile acids and other (post-)transcriptional modulators? Clin Rev Allergy Immunol 2009;36:52-61.

44. Fickert P, Hirschfield GM, Denk G, et al. norUrsodeoxycholic acid improves cholestasis in primary sclerosing cholangitis. J Hepatol 2017;67:549-58. 